\title{
Orthodontic and Periodontic Concoction: A Review
}

\author{
Dr Sumita Mishra, Dr Smruti Bhusan Nanda² \\ ISenior Lecturer, ${ }^{2}$ Reader, Dept of Orthodontics, \\ Institute of Dental Sciences, Bhubaneswar, India
}

Correspondence: Dr Sumita Mishra; Email: sumita.mitali@gmail.com

\section{ABSTRACT}

The article emphasizes on the need for amalgamation of orthodontic and periodontic treatment. The need arises when we need to respect the integrity of the periodontal apparatus enhancing the importance of oral health. The beauty of orthodontics in the long run is envisaged by the surrounding sound environment of gingiva. Orthodontic treatment is carried out in various stages: initial leveling and alignment, space closure and finishing, detailing respecting the force requirements, mechanics; different wires are being used to fit the various clinical situations. The periodontium needs to be in harmony to yield a successful orthodontic treatment outcome.

Keywords: orthodontics, ortho-perio relationship, peridontics

\section{INTRODUCTION}

Much influenced by the thought provoking article by Dr. Vincent G. Kokich, the catch to manage orthodontic patients who are periodontally compromised has immensely increased.' Interdisciplinary orthodontics plays a vital role in day-to-day management of patients. ${ }^{2}$ Periodontium serves as the medium by which orthodontists are able to carry out various types of orthodontic tooth movement. Periodontal health becomes questionable when we do not respect its integrity. ${ }^{3}$ There are various cases which report to the orthodontists for management of malaligned teeth with high risk to the periodontium. These aspects need to be carefully managed and amalgamated with orthodontic treatment.

Certain protocols need to be followed while treating cases that pose high risk to the periodontium. The primary objective

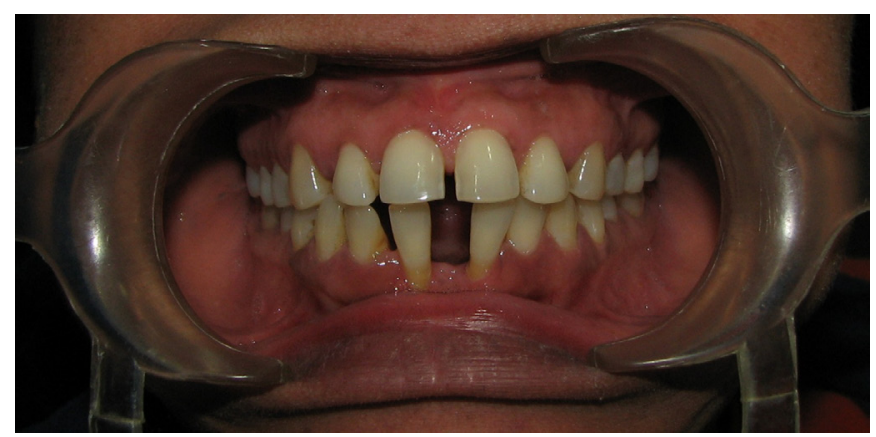

Figure 1: Pre-treatment; compromised periodontal health in lower anterior region of periodontal therapy is to restore and maintain health and integrity of the attachment apparatus of the teeth. The treatment is complicated in patients with periodontally compromised teeth. Thus, orthodontic treatment is necessary as an adjunct in solving these problems.

\section{CASE STUDIES}

Various patients seek quick treatment for correction of malaligned teeth. Such patients need to be patiently handled and explained about all aspects that would affect their periodontal health and then decide on the treatment. Here are few cases where orthodontics and periodontics get amalgamated.

The first case is presented with a chief complaint of gap in upper and lower front teeth region. The major concern in the case was the age of the patient and the periodontal health

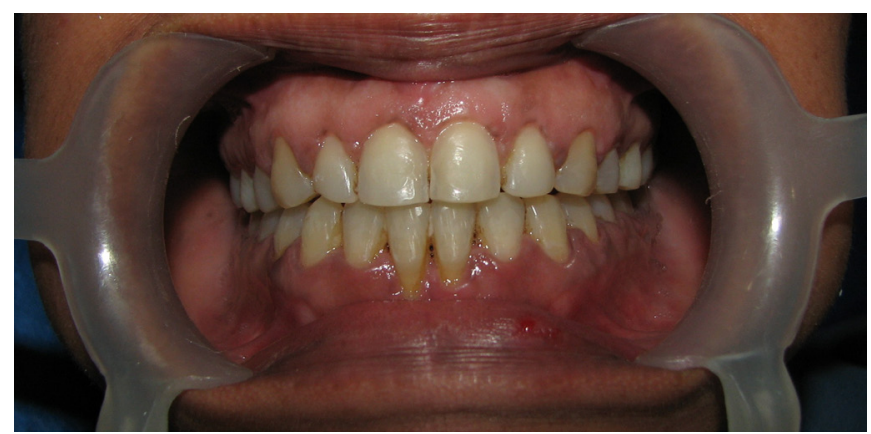

Figure 2: Post-treatment; improved periodontal status 


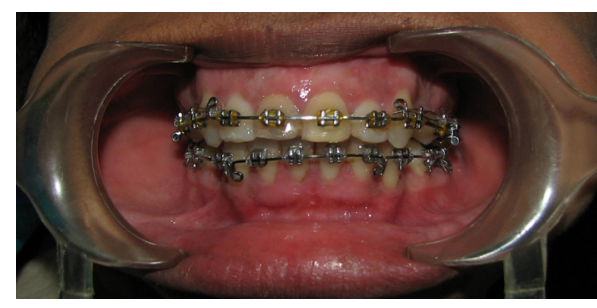

Figure 3: Conventional orthodontic treatment; extraction of all first premolars

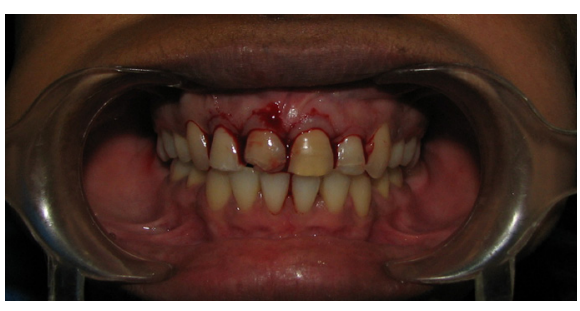

Figure 4: Periodontal incision

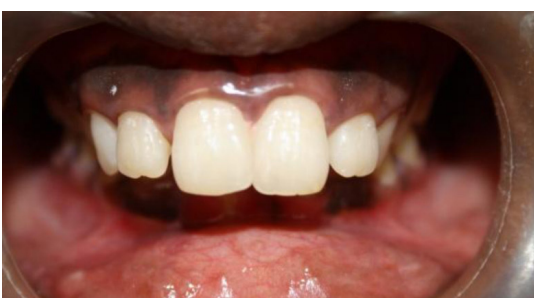

Figure 5: Pre-treatment; Class II Div I malocclusion of the lower anterior tooth region as shown in Figure 1. The orthodontic treatment was started using 0.0175 coaxial wires, the wire chosen has low force load deflection rate compared to the Nickel Titanium wires (NiTi). ${ }^{4}$ Attempts to reduce the load deflection ratio led to the introduction of multistranded steel wires, nonsuperelastic or martensitic stabilized NiTi wires, superelastic or austenitic active NiTi wires, and true shape memory or martensitic active wires for initial alignment. ${ }^{5}$ The patient was put on rigorous oral hygiene maintenance therapy. It included proper toothbrushing and mouth rinsing. Initial leveling and alignment was carried out. During the course of treatment, elastomeric chains were avoided for space closure to prevent any further periodontal insult. Instead, ligature wires in 'figure 8' motion are preferred. Post treatment showed improvement of the periodontal status as evident in Figure 2.

The second case presented with the chief complaint of forwardly placed upper and lower front teeth. The patient complained of excessive gingival display. It warranted for surgical treatment but the patient was not interested. All first premolars were extracted using conventional orthodontic procedure as shown in Figure 3. Periodontally, incisions were given as shown in Figure 4. These incisions were made to reduce the gingival display which was much evident after orthodontic treatment.

The third case presented with the chief complaint of forwardly placed upper jaw and irregularly placed lower front teeth. It was diagnosed as a Class II Div I case with severe lower anterior crowding (Figure 5 \& 6). The lower right lateral incisor exhibited gingival recession (Figure 7). Proceeding the case without periodontal concern would not yield proper result. After initial leveling and alignment with 0.0175 coaxial wires and further stabilization with rectangular stainless steel wire, the same incisor was operated for a flap as shown in Figure 8.

\section{DISCUSSION}

According to Proffit, ${ }^{6}$ and Bennett and McLaughlin, ${ }^{7}$ the first phase of orthodontic treatment deals with alignment and correction of vertical and horizontal discrepancies by leveling out the arches, and the initial aligning wires should apply light continuous force. Conventionally, round wires are used for alignment because tightly fitting resilient rectangular archwires produce back-and forth movement of the root apices as the teeth move into alignment. ${ }^{8}$ Here, we used coaxial wires to maintain low force load deflection rate.

Multistranded archwires to gain mechanical advantages such as increased flexibility and a reduced load deflection rate has been successfully attempted with the use of stainless steel.9-12 The same concept was applied in the case of superelastic NiTi; ${ }^{13}$ this led to the introduction of Supercable, a seven-stranded round coaxial superelastic NiTi archwire. ${ }^{13-15}$ According to the manufacturers, this wire does not take a set after bending. Laboratory tests seem to suggest that these wires exert only $36-70 \%$ of the force of solid NiTi wires. These wires deliver force levels at a constant force decay rate avoiding any periodontal insult. When not taken care of, would lead to tooth mobility and loss of attachment. When the chances of 'loss of tooth vitality' are apprehended, intentional root canal treatment can be performed and orthodontic treatment can be carried thereafter successfully.

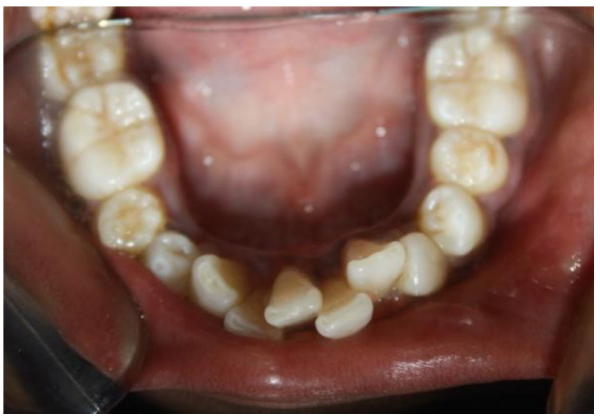

Figure 6: Lower anterior crowding

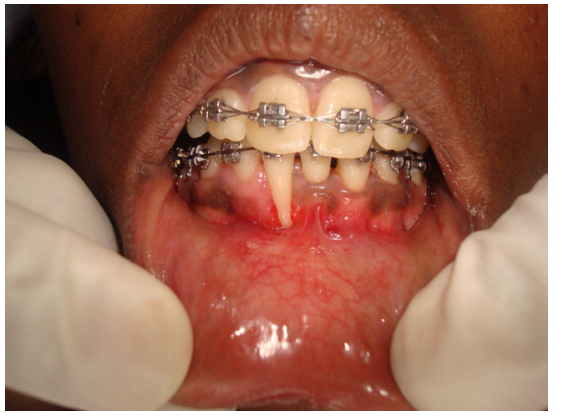

Figure 7: Gingival recession

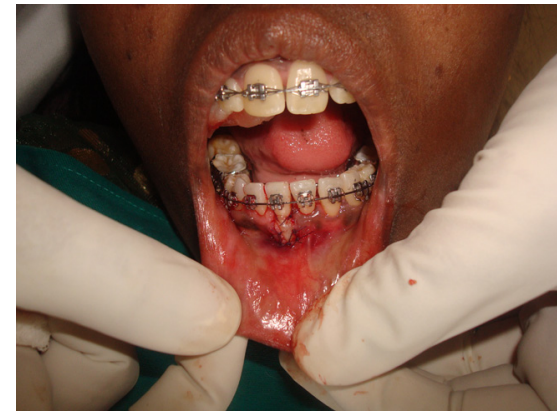

Figure 8: Flap surgery 
There are various periodontal considerations that need to be addressed. Crowding creates inaccessible areas between and around the teeth. If the teeth receive occlusal load non-axially, lateral force is created which is unfavorable. There are also untoward occlusal forces in excess of the reparative capacity of the attachment apparatus. This can be primary (where changes are induced by occlusal forces in excess of normal masticatory function) or secondary (where changes are induced by occlusal forces produced by normal masticatory function on teeth with decreased attachment apparatus).

Pathogens associated with periodontal disease tend to increase in number during fixed orthodontic treatment. Orthodontic mechanics using light forces and bonded brackets rather than bands are recommended if there are concerns with oral hygiene or if there is reduced periodontal attachment. Appliance design should be as simple as possible with appliances that will collect less plaque and not irritate gingival tissues.

Periodontal considerations during orthodontic treatment include:

1. Pericision; to reduce the rebound of elastic fibres in the supracrestal tissue where we anticipate relapse of severely rotated teeth. Other pericision techniques include central papilla incision, reverse bevel CSF and electro-surgery techniques.

2. Frenectomy; in cases of diastema with a large/broad frenum. Other techniques include simple excision, Z-plasty and laser removal.

3. Mucogingival considerations; It must be remembered that there is a biologic and genetically determined envelope for bone and mucogingival margin position, hence there is potential for gingival recession when teeth are moved outside this envelope. This movement can be as a result of anterior or posterior over expansion. Treatment planning must take into account the possibility of recession, connective tissue loss and the implications regarding stability.

4. Gingival grafting; Mucogingival surgery may be indicated in situations where a tooth that is planned for orthodontic movement has thin facial gingiva. By increasing the thickness of the covering tissue, the risk of gingival recession during or following the orthodontic therapy should be reduced. Numerous grafting techniques have been advocated including free gingival grafts and interpositional pedicle grafts. ${ }^{16}$ The free gingival graft (FGG) provides increased thickness of tissue and increased width of keratinised tissue. Pedicle grafts (laterally repositioned flap, LRF and coronally repositioned flap, CRF) are dependent on the presence of adequate width of keratinized tissue either adjacent or apical to the defect. Both the LRF and CRF provide acceptable results in terms of tissue augmentation, aesthetics and root coverage.

\section{CONCLUSION}

During recent times, the number of adults seeking orthodontic treatment has increased significantly. Treatment of periodontally compromised adults with a skeletal Class II occlusion has always been a clinical challenge with alveolar bone loss. In addition, the loss of attachment will relocate the center of resistance further apically, which affects the moment generated by a respective force. Thus, biomechanics in a periodontally compromised patient differs from a healthy one, requiring lighter forces and relatively larger moments.

Profound improvement in treatment outcome with an improved functionally stable occlusion and a well maintained periodontium can be achieved by the combined therapy of orthodontics and periodontics. 


\section{REFERENCES}

1. Vincent G. Kokich. The key to adult orthodontics. Am J Orthod Dentofacial Orthop 201 1; 139:289.

2. David L. Turpin. Interdisciplinary care leads the way. Am J Orthod Dentofacial Orthop 2001; 119:235.

3. Teresa P, Manuel N, Celia A. Multidisciplinary management including periodontics, orthodontics, implants, and prosthetics for an adult. Am J Orthod Dentofacial Orthop 2012; 142:235-45.

4. Dalstra M, Melsen B. Does the transition temperature of Cu-NiTi archwires affect the amount of tooth movement during alignment? Orthod Craniofac Res. 2004; 7:21-5.

5. Brantley WA. Orthodontic wires. In: Brantley WA, Eliades T,eds. Orthodontic Materials: Scientific and Clinical Aspects. Stuttgart, Germany: Thieme; 2001:91-9.

6. Proffit WR, Fields HW, Sarver DM. Contemporary Orthodontics.4th ed. St Louis, Mo: Mosby; 2007:551-2.

7. Bennett JC, McLaughlin RP. Orthodontic Treatment Mechanics and the Preadjusted Appliance. 1st ed. Prescott, Ariz: Wolfe Publishing; 1993:89.

8. Proffit WR, Fields HW, Sarver DM. Contemporary Orthodontics, 4th ed. St Louis, Mo: Mosby; 2007:553.

9. Riley M, Bearn DR. A systemic review of clinical trials of aligning archwires. J Orthod. 2009; 36:42-51.

10. Pandis N, Polychronopoulou A, Eliades T. Alleviation of mandibular anterior crowding with copper-nickel-titanium vs nickel-titanium wires: a double-blind randomized control trial. Am J Orthod Dentofacial Orthop. 2009; 136:151-7.

11. Ong E, Ho C, Miles P. Alignment efficiency and discomfort of three orthodontic archwire sequences: A randomized clinical trial. J Orthod. $2011 ; 38: 32-9$.

12. Kusy RP, Dilley GJ. Elastic modulus of a triple-stranded stainless steel archwire via three- and four-point bending. J Dent Res. 1984; $36: 1232-40$.

13. Kusy RP, Stevens LE. Triple-stranded stainless steel wires: Evaluation of mechanical properties and comparison with titanium alloy alternatives. Angle Orthod. 1987; 57:18-32.

14. Rucker BK, Kusy RP. Theoretical investigation of elastic flexural properties for multistranded orthodontic archwires. J Biomed Mater Res. 2002; 62:338-49.

15. Rucker BK, Kusy RP. Elastic flexural properties of multistranded stainless steel versus conventional nickel titanium archwires. Angle Orthod 2002; 72:302-9.

16. Lindhe J. Clinical Periodontology, 2nd Ed, Munksgaard, Copenhagen; 1992. 